\title{
Capacidades estatales y cambio en las políticas públicas: el caso de Uruguay bajo los gobiernos del Frente Amplio (2005-2015)
}

\author{
Nicolas Bentancur \\ Doctor en Ciencias Sociales (Universidad de Buenos Aires) \\ Docente- Investigador de la Universidad de la República - Uruguay \\ Montevideo, Uruguay \\ nicobent63@gmail.com \\ Jose Miguel Busquets \\ Doctor en Ciencia Política (Cándido Mendes) \\ Docente-Investigador de la Universidad de la República \\ Montevideo, Uruguay \\ busquets@chasque.net
}

El artículo tiene como propósito indagar sobre el cambio en las políticas públicas en Uruguay durante los dos primeros períodos de gobierno de un partido de izquierda, el Frente Amplio (2005-2015). Para ello se analiza en qué medida cambiaron un conjunto de políticas, y las asociaciones existentes entre las diferentes capacidades estatales desarrolladas y el tipo de cambio implementado en cada una de ellas. Tras una indagación que reposa en estudios previos, en una consulta a expertos y en la aplicación de la metodología comparativa cualitativa (QCA), se concluye que en ese país la llegada al poder ejecutivo del Frente Amplio supuso transformaciones significativas en la orientación de las políticas, aunque con desigual intensidad en distintos puntos del tejido público. Las capacidades estatales mostraron ser variables relevantes para contribuir a comprender esas transformaciones y diferencias, resultando que en los sectores en que dichas capacidades fueron fuertes, fue posible propiciar transformaciones de porte; $y$ donde no lo fueron, se registraron cambios de menor entidad.

Palabras clave: políticas públicas, gobiernos de izquierda, capacidades estatales, cambio en las políticas públicas, Uruguay. 


\section{Introducción}

En el año 2005 accedió al gobierno nacional, por primera vez en la historia de Uruguay, un partido político de izquierda. El Frente Amplio fue fundado en el año 1971 con el propósito de superar la grave crisis política que por entonces sufría el país, pero también para impulsar reformas estructurales en el Estado, la economía y la sociedad. A la primera presidencia de Tabaré Vázquez entre los años 2005 y 2010 le sucedió la de José Mujica, entre 2010 y 2015.

Luego de diez años de gestión del Estado por parte de este partido político, es pertinente interrogarse en qué medida las diferentes políticas públicas implementadas en ese período han supuesto cambios de relevancia en las orientaciones imperantes hasta el momento de su ascenso al gobierno, lo que conduce a reflexionar sobre el grado de innovación o tipos de cambio materializados.Y más específicamente, sobre las eventuales relaciones entre las capacidades estatales y esos cambios, en tanto dichas capacidades suelen identificarse en la literatura especializada como una de las variables claves para explicarlos.

El caso es de particular interés analítico por tratarse de la primera gestión de gobierno -durante dos períodos consecutivos - de un partido de izquierda en Uruguay: el Frente Amplio. ¿En qué medida ello generó novedades en las políticas públicas impulsadas? ¿De qué capacidades estatales se dispuso para promover esos cambios? ¿Difirieron esas capacidades en las distintas arenas de políticas?Y en caso de que haya sido así, ¿dieron lugar a transformaciones también diversas?

A los efectos de desarrollar nuestro argumento utilizamos como insumo fundamental los estudios sectoriales contenidos en el trabajo "El decenio progresista. Las políticas públicas de Vázquez a Mujica" (Bentancur; Busquets, 2016). Los trece casos analizados tienen una homogeneidad externa de carácter político-institucional, por corresponder a los dos primeros períodos de gobierno del Frente Amplio en Uruguay; y una heterogeneidad interna dada por las distintas áreas de políticas estudiadas. Asimismo, realizamos un análisis transversal del producto colectivo, y adicionamos otros estudios de investigadores, implementamos una encuesta a expertos en políticas públicas sobre el tipo de cambio y las capacidades estatales $^{1} \mathrm{y}$, finalmente, utilizamos la metodología comparativa cualitativa (QCA). ${ }^{2}$

Luego de esta introducción, el texto se compone de cuatro apartados. El segundo se dedica a las capacidades estatales, presentando el marco conceptual y su aplicación al caso de los gobiernos del Frente Amplio en Uruguay. El tercero da cuenta de los cambios en las políticas públicas procesados en el período 2005-2015. Posteriormente, en el cuarto apartado se analiza la correlación existente en nuestro caso entre ambas variables: capacidades estatales y cambios en las distintas políticas sectoriales. Por último, el quinto apartado se dedica a las conclusiones derivadas de nuestra indagación.

1 La encuesta a expertos fue realizada a través de un formulario con un conjunto de preguntas cerradas. A partir de la consideración de los expertos definimos el valor de las variables que se referirán más adelante. 


\section{Las capacidades estatales en los gobiernos del frente amplio}

Pueden identificarse varias dimensiones en las estrategias de los gobiernos del Frente Amplio para gestionar sus políticas públicas. Entre ellas, un tipo de intermediación de intereses que se corresponde con el formato pluralista pero reconoce incrustaciones neocorporativas (Schmitter, 1977; Lanzaro, 1986); un rol destacado de los partidos en el policymaking (Caetano et al, 1987; García Pelayo, 1986); la apelación a instrumentos tales como "debates" o "diálogos" sociales (Pereira, 2015); y la nítida predominancia del juego político sobre la racionalidad técnica, aunque con diferencias según la arena de políticas que se considere (Campbell; Pedersen, 2011; Garcé, 2014).

Aquí haremos foco en otra variable: las capacidades estatales, que en estudios anteriores (Franco Chuaire; Scartascini, 2014; Pires; Gomide, 2016; Repetto, 2004; Soifer, 2012) se han asociado con las orientaciones de las políticas públicas en distintos países de América Latina. La relevancia que en esta línea analítica se otorga al concepto queda de manifiesto en asertos como el siguiente:

La relevancia de las capacidades estatales para incidir efectivamente en la calidad de las políticas públicas ha sido constatada en múltiples estudios empíricos. Entre ellos, los producidos por el BID para trece países de América Latina, en los que se ha concluido que "... las capacidades gubernamentales son fundamentales para contar con políticas públicas con mejores características". (Franco Chuayre; Scartascini, 2014).

Nos adscribimos a estos enfoques, que sustentan la pertinencia de analizar las asociaciones y mecanismos causales que operan en el caso en estudio entre capacidades estatales, por un lado, y la magnitud de los cambios en las políticas públicas, por otro.

En este punto corresponde precisar el concepto de capacidades estatales que emplearemos. En primer lugar, y como señala Iazzetta (2012), no deben confundirse capacidades estatales con capacidades de gobierno. Mientras estas últimas refieren a acciones concretas impulsadas en cierto momento por un gobierno dado, las capacidades estatales se sustentan en períodos más largos y remiten a la construcción institucional del aparato estatal.

Es menester señalar, asimismo, que el concepto tiene una larga historia en la que se ha atendido a dimensiones diversas. En algunos autores, en la noción de capacidad estatal predomina la dimensión fiscal (Tilly, 1992), en otros autores predomina la dimensión burocrática o administrativa (Mann, 1984, 2008; Oszlak, 1980; Geddes, 1994; Evans; Rauch, 1999) y en otros la dimensión de coerción estatal (Hendrix, 2010). En los abordajes tradicionales, los énfasis daban mucha importancia a la dimensión burocrática, mientras que en los enfoques más contemporáneos se establece una relación entre la capacidad estatal y la gobernanza. Actualmente, la literatura sobre capacidades estatales o del Estado puede ser analizada recurriendo a una diversidad de definiciones (Altman; Luna, 2012; Edigheji, 2010; Evans; Rauch, 1999; Pires; Gomide, 2016). 
Como señalan estos últimos autores, y sin perjuicio de las variantes interpretativas existentes, en la literatura especializada las capacidades de los Estados se suelen asociar con sus habilidades y competencias para establecer sus objetivos y realizarlos, ligando así el término con las preocupaciones por la gobernabilidad. A continuación, identifican dos dimensiones que consideramos de recibo:

a) las técnico-administrativas involucran las capacidades derivadas de la existencia y funcionamiento de burocracias competentes y profesionalizadas, dotadas de recursos organizacionales, financieros y tecnológicos necesarios para conducir las acciones del gobierno de forma coordinada; b) las políticorelacionales se asocian a las habilidades y procedimientos de inclusión de los múltiples actores (sociales, económicos y políticos) de forma articulada en los procesos de políticas públicas, buscando la construcción de consensos mínimos y coaliciones de apoyo a las políticas gubernamentales. La primera dimensión puede asociarse a las nociones de eficiencia y eficacia, y la segunda está relacionada con las ideas de legitimidad, aprendizaje e innovación en las acciones de los gobiernos. (Pires; Gomide, 2016, p. 127). ${ }^{3}$

Los mismos autores proponen que las capacidades técnico administrativas pueden ser analizadas según tres aspectos: a) la presencia de las burocracias gubernamentales profesionalizadas (ORGA); b) el funcionamiento de mecanismos de coordinación intra-gubernamental (COOR) y c) la existencia de procedimientos de monitoreo de la implementación (MONI). Por su parte, las capacidades político-administrativas pueden ser evaluadas también por tres aspectos: a) interacciones institucionalizadas entre actores burocráticos y agentes políticos (parlamentarios de diversos partidos) (APOLI); b) la existencia y el funcionamiento de mecanismos de participación social (PART) y c) la presencia de fiscalización realizada por agencias de control (CONT).

Aplicamos el marco analítico recién expuesto a un relevamiento de los juicios de quince académicos expertos en trece arenas de políticas públicas del caso uruguayo. De esa forma obtuvimos los siguientes resultados referidos a las capacidades técnico-administrativas (cuadro 1), y político-relacionales (cuadro 2), en los que “0” indica ausencia de una variable de la capacidad estatal analizada, y "1" su presencia.

Cuadro 1. Capacidades técnico-administrativas en sectores de políticas, Uruguay (2005-2015)

\begin{tabular}{|c|c|c|c|}
\hline & ORGA & COOR & MONI \\
\hline Educación Superior & 1 & 1 & 0 \\
\hline Tributaria & 1 & 1 & 1 \\
\hline Sanitaria & 1 & 1 & 1 \\
\hline Laboral & 1 & 1 & 1 \\
\hline Agropecuaria & 1 & 1 & 0 \\
\hline Género & 1 & 1 & 1 \\
\hline
\end{tabular}




\begin{tabular}{|c|c|c|c|}
\hline Asistencia Social & 1 & 1 & 1 \\
\hline Seguridad Pública & 1 & 1 & 1 \\
\hline Política exterior & 0 & 1 & 0 \\
\hline Seguridad Social & 1 & 1 & 0 \\
\hline Defensa & 0 & 1 & 1 \\
\hline Vivienda & 1 & $+/-$ & 0 \\
\hline Educación & 0 & 1 & 1 \\
\hline
\end{tabular}

Fuente: Elaboración propia, según encuesta a expertos, 2017.

Cuadro 2. Capacidades político-relacionales administrativas en sectores de políticas, Uruguay 2005-2015

\begin{tabular}{|c|c|c|c|}
\hline & APOL & PART & CONT \\
\hline Educación Superior & 1 & 1 & 1 \\
\hline Tributaria & 0 & 1 & 1 \\
\hline Sanitaria & 0 & 1 & 1 \\
\hline Laboral & 1 & 1 & 1 \\
\hline Agropecuaria & 1 & 1 & 0 \\
\hline Género & 0 & 1 & 1 \\
\hline Asistencia Social & 0 & 1 & 0 \\
\hline Seguridad Pública & 1 & 1 & 1 \\
\hline Exterior & 1 & 1 & 0 \\
\hline Seguridad Social & 1 & 0 & 1 \\
\hline Defensa & 0 & 0 & 0 \\
\hline Vivienda & 0 & 1 & \\
\hline Educación & 0 & & 1 \\
\hline
\end{tabular}

Fuente: Elaboración propia, según encuesta a expertos, 2017.

De ambos cuadros se desprende que en el conjunto de las trece políticas públicas analizadas, las capacidades estatales técnico-administrativas del período resultaron más frecuentes que las capacidades político-relacionales. Dentro de las técnico-administrativas, la presencia de las burocracias gubernamentales profesionalizadas (ORGA) y el funcionamiento de mecanismos de coordinación intra-gubernamental (COOR) fueron las más relevantes. En tanto, dentro de las político-administrativas la existencia y el funcionamiento de mecanismos de participación social (PART), fue la variable que alcanzó el mayor número de caracterizaciones positivas en el conjunto de políticas públicas analizadas. 


\section{Innovaciones, ajustes y acumulaciones en las políticas públicas uruguayas del período 2005-2015}

En la medida que nos propusimos estimar el grado de innovación introducido en el decenio posterior sobre las políticas en curso al año 2005, es menester presentar previamente algunas referencias teóricas a la cuestión del cambio en las políticas públicas. Como ha sostenido Stewart, “comprender por qué, cuándo y cómo las políticas públicas cambian (o no) es uno de los problemas de investigación centrales de las ciencias de las políticas públicas" (Stewart, 2006, p. 184).

Los estudios académicos sobre el punto han asumido que las políticas tienden a la continuidad, y que los cambios que experimentan son, por lo general, graduales. Sobre esta asunción se fundó la escuela del incrementalismo, que sostiene que las innovaciones son normalmente marginales en relación a los lineamientos de políticas precedentes, por dos razones: a) derivan del acuerdo entre partidarios con distintos intereses y valores, lo que excluye alternativas radicales; y b) se seleccionan mediante métodos que procuran reducir la complejidad, acotando el análisis a las diferencias incrementales entre alternativas cercanas (Lindblom, 1992). Paralelamente, otros cuerpos de ideas se ocuparon de las circunstancias en las que los cambios significan una ruptura radical con las políticas precedentes, atribuyéndolos a la verificación de ciertas coyunturas críticas en los sistemas políticos, que resultan aptas para interrumpir la dinámica inercial de las instituciones (Capoccia; Kelemen, 2007; Collier; Collier, 1991; Pierson, 2000; Weir; Skocpol, 1993). Pero en todo caso estos cambios paradigmáticos han sido considerados como excepciones al flujo previsible y relativamente constante de las corrientes de políticas.

Sin embargo, indagaciones recientes han sugerido que es frecuente que pequeños cambios de naturaleza incremental originen, por acumulación y permanencia en el tiempo, transformaciones institucionales significativas. Esas indagaciones han distinguido la dimensión procedimental, por lo general gradualista, de los resultados de ese proceso, que en ocasiones genera discontinuidades de relieve (Baumgartner et al, 2006; Pierson, 2004; Streek; Thelen, 2005). Por ende, no puede colegirse en todos los casos que la hechura de políticas de naturaleza incremental conducirá a una reproducción meramente adaptativa de las precedentes, de la misma forma que las estrategias de shock no garantizan transformaciones profundas y duraderas.

Si el cambio gradual es relevante y objeto de interés, es menester entonces disponer de tipologías para mensurarlo debidamente. Existen múltiples propuestas con esa finalidad (Cruz Rubio, 2012; Durant; Diehl, 1989; Hall, 1989,1993; Hogwood; Peters, 1982; Howlett; Cashore, 2009; Mahoney; Thelen, 2010; Sabatier;Weible, 2010; Streek; Thelen, 2005).

Aquí aplicaremos una de las categorizaciones de mayor recibo en la literatura académica, elaborada por Peter Hall (1989, 1993). La misma distingue cambios de primer orden dirigidos a calibrar instrumentos de políticas existentes que perviven; de segundo orden, consistentes en modificaciones en los instrumentos empleados; y de tercer orden, representativos de ajustes paradigmáticos en los fines de las políticas. 
A continuación (Cuadro 3) presentamos de manera sintética los lineamientos sobresalientes de las trece políticas públicas estudiadas. A posteriori (Cuadro 4), detallamos el grado de cambio o tipo de innovaciones encontradas por los expertos en las trece políticas públicas seleccionadas, tomando como criterio la tipología recién referida.

Cuadro 3. Principales lineamientos de políticas públicas, Uruguay 2005-2015

\begin{tabular}{|c|c|}
\hline Arena de Política & Lineamientos principales \\
\hline Educación Superior & $\begin{array}{l}\text { Reforma de la Universidad de la República. Creación de Uni- } \\
\text { versidad Tecnológica. Expansión de la educación superior en el } \\
\text { territorio. Agencia Nacional de Innovación e Investigación. }\end{array}$ \\
\hline Tributaria & $\begin{array}{l}\text { Reforma tributaria, con introducción del Impuesto a la Renta } \\
\text { de las Personas Físicas. Flexibilización del "secreto bancario" }\end{array}$ \\
\hline Sanitaria & $\begin{array}{l}\text { Creación de Sistema Nacional Integrado de Salud. Des- } \\
\text { centralización de Servicios de Salud del Estado }\end{array}$ \\
\hline Laboral & $\begin{array}{l}\text { Reinstalación de "Consejo de Salarios", organismo tripartito que } \\
\text { rige las relaciones laborales. Leyes de protección laboral y sindical }\end{array}$ \\
\hline Agropecuaria & $\begin{array}{l}\text { Articulación territorial entre actores del agro. Inclusión de } \\
\text { productores familiares en cadenas agro-industriales. Fo- } \\
\text { mento de la innovación tecnológica aplicada al agro }\end{array}$ \\
\hline Género & $\begin{array}{l}\text { Ley de Igualdad de Oportunidades y Derechos. Des- } \\
\text { penalización del aborto. Matrimonio igualitario }\end{array}$ \\
\hline Asistencia Social & $\begin{array}{l}\text { Creación de Ministerio de Desarrollo So- } \\
\text { cial. Ajustes en prestaciones universales y expan- } \\
\text { sión de prestaciones focalizadas preexistentes }\end{array}$ \\
\hline Seguridad Pública & $\begin{array}{l}\text { Continuación de políticas precedentes ("Leyes de Segu- } \\
\text { ridad Ciudadana"), con otros énfasis y adaptaciones }\end{array}$ \\
\hline Exterior & $\begin{array}{l}\text { Pervivencia de políticas de integración regio- } \\
\text { nal, pero pasando de un "regionalismo abierto" a } \\
\text { una "integración regional/multilateralismo" }\end{array}$ \\
\hline Seguridad Social & $\begin{array}{l}\text { Permanencia de reforma estructural de los años 90s (sistema ju- } \\
\text { bilatorio mixto público-privado), con ampliación de la cobertura }\end{array}$ \\
\hline Defensa & $\begin{array}{l}\text { Ley Marco de Defensa Nacional, solo parcialmente implemen- } \\
\text { tada. Estructura militar y estrategia de defensa no modificadas }\end{array}$ \\
\hline Vivienda & $\begin{array}{l}\text { Sistema de mercado y centralidad de actores privados. Sub- } \\
\text { sidios estatales a grupos vulnerables (Plan Juntos) y exo- } \\
\text { neraciones fiscales para "viviendas de interés social" }\end{array}$ \\
\hline Educación & $\begin{array}{l}\text { Principales reformas postergadas por restricciones ins- } \\
\text { titucionales y oposición de sindicatos docentes }\end{array}$ \\
\hline
\end{tabular}

Fuente: elaboración propia, 2017. 
Cuadro 4. Tipología de las políticas públicas, Uruguay 2005-2015

\begin{tabular}{|c|c|}
\hline Arena de Política & Tipo de cambio \\
\hline Educación Superior & Tercer orden \\
\hline Tributaria & Tercer orden \\
\hline Sanitaria & Tercer orden \\
\hline Laboral & Tercer orden \\
\hline Agropecuaria & Tercer orden \\
\hline Género & Segundo orden \\
\hline Asistencia Social & Segundo orden \\
\hline Seguridad Pública & Segundo orden \\
\hline Exterior & Segundo orden \\
\hline Seguridad Social & Segundo orden \\
\hline Defensa & Primer orden \\
\hline Vivienda & Primer orden \\
\hline Educación & Primer orden \\
\hline
\end{tabular}

Fuente: elaboración propia, en base a encuesta a expertos, 2017

Se distinguen así tres bloques de políticas en relación al grado de transformación impuesto. El primero, el de "cambios de tercer orden" contempla en primer lugar creaciones institucionales claves en el terreno de la educación superior, incluyendo una nueva universidad pública (la segunda del país), la instalación de Centros Regionales de la Universidad de la República en el interior del país, y el establecimiento de un organismo gubernamental destinado a promover el desarrollo científico y tecnológico (la Agencia Nacional de Investigación e Innovación). Asimismo, también como "cambios de tercer orden" se comprenden otras cuatro arenas de políticas, en las que los gobiernos del Frente Amplio imprimieron transformaciones sustantivas en relación al estatus precedente. Valgan como ejemplo de las mismas, una reforma tributaria que reconfiguró el sistema -incluyendo la introducción de un Impuesto a la Renta de las Personas Físicas-, la instauración de un sistema nacional integrado de salud y la aprobación de un amplio conjunto de leyes de protección laboral, conjuntamente con la reinstauración de los Consejos de Salarios como pieza clave de la regulación del trabajo. Finalmente, corresponde también destacar la magnitud en los cambios en las políticas agropecuarias, alineadas a un modelo de desarrollo multidimensional y sustentadas en procesos de innovación tecnológica aplicados al sector.

El segundo bloque, que agrupa "cambios de segundo orden", representa la reorientación parcial de políticas precedentes. Así, se continúa con la estrategia de políticas focalizadas para atender la cuestión social que habían sido inauguradas por los partidos tradicionales que precedieron al Frente Amplio en el gobierno, pero el foco se amplía para algunas prestaciones y se ajustan otras de naturaleza universal, al tiempo que se instituye un Ministerio específico. En materia de seguridad pública se ensayan nuevas estrategias para enfrentar la criminalidad, dentro de un modelo orientado a la comunidad 
o de servicio público, con antecedentes en los años anteriores. En relación a la política exterior, se conjugaron el regionalismo precedente con un multilateralismo enfocado en los BRICS, mientras que en seguridad social ha pervivido la reforma estructural de los años noventa, que instauró un régimen mixto público-privado para las pensiones, pero se han procesado ajustes relevantes por la vía de diálogos político-sociales.

Sobre los sectores del último bloque de políticas se operó en el último decenio incorporando nuevos programas e instrumentos, que no se dirigieron a sustituir o modificar sustancialmente los empleados hasta entonces, por lo que constituyen cambios de primer orden. Dentro de este conjunto pueden identificarse matices de importancia. En el extremo inferior, las políticas de vivienda y de educación exhibieron muy escasas notas de novedad. Por otro lado, en defensa se ensayaron algunos énfasis que permiten separar a las políticas del período de las formuladas previamente por los partidos tradicionales (la Ley Marco de Defensa Nacional, sin embargo implementada solo parcialmente).

Si se contempla la impronta transformadora del Frente Amplio en el marco de las arenas de políticas seleccionadas en toda su complejidad y diversidad, debe concluirse que se caracteriza por una gran dispersión, entre registros constituyentes o re-configuradores en algunas de ellas, y meramente adaptativos en otras. Por un lado, se establecieron nuevas institucionalidades y reglas de juego para sectores y temáticas de gran relevancia política y social. Por otro, algunas de las reformas características de las políticas de los años noventa fueron asimiladas (seguridad social) o no se hallaron fórmulas para su sustitución (educación).Y en amplios espacios, se operó con la estrategia de incorporar nuevas capas de políticas sobre las ya existentes (Mahoney; Thelen, 2010).

\section{Comparando las asociaciones entre capacidades estatales y tipo de cambio o innovaciones}

Tomando en cuenta las dimensiones sobre capacidades estatales presentadas en el apartado 2, el tipo de cambio registrado en las políticas según se consignó en el apartado 3, y los resultados de la encuesta a expertos, es posible presentar ahora todas las configuraciones para la variable tipo de cambio o innovación (INN). Para desarrollar esta argumentación optamos por cotejar los casos con el tipo de cambio de tercer orden y de primer orden, excluyendo los casos que exhibieron cambios de segundo orden, con el propósito de dicotomizar la variable.

Las explicaciones se desarrollan a partir de las tres diferentes capacidades estatales técnico-administrativas y las tres capacidades político-relacionales, como variables causales (Cuadro 5). Los argumentos presentados no tienen contradicciones que tengan que ser resueltas, o sea no hay dos argumentos iguales para diferentes resultados, por lo cual seleccionamos las combinaciones con resultado $\mathrm{R}=1=$ cambios de tercer orden para aplicar el procedimiento de simplificación de la matriz booleana. 
Cuadro 5. Configuraciones de casos para la variable tipo de cambio o innovación

\begin{tabular}{|c|c|c|c|c|c|c|c|}
\hline & ORGA & COOR & MONI & APOL & PART & CONT & INN \\
\hline $\begin{array}{c}\text { Educación } \\
\text { Superior }\end{array}$ & 1 & 1 & 0 & 1 & 1 & 0 & 1 \\
\hline Tributaria & 1 & 1 & 1 & 0 & 1 & 1 & 1 \\
\hline Sanitaria & 1 & 1 & 1 & 0 & 1 & 1 & 1 \\
\hline Laboral & 1 & 1 & 1 & 1 & 1 & 1 & 1 \\
\hline $\begin{array}{c}\text { Agrope- } \\
\text { cuaria }\end{array}$ & 1 & 1 & 1 & 1 & 1 & 1 & 1 \\
\hline Defensa & 0 & 1 & 0 & 0 & 0 & 0 & 0 \\
\hline Vivienda & 1 & 0 & 1 & 0 & 0 & 1 & 0 \\
\hline Educación & 0 & 1 & 0 & 0 & 1 & 0 & 0 \\
\hline
\end{tabular}

Fuente: Elaboración propia, según encuesta a expertos, 2017.

Cuadro 6. Configuraciones posibles de casos para la variable tipo de cambio o innovación

\begin{tabular}{|c|c|c|c|c|c|c|c|}
\hline & ORGA & COOR & MONI & APOL & PART & CONT & INN \\
\hline $\begin{array}{c}\text { Educación } \\
\text { Superior }\end{array}$ & 1 & 1 & 0 & 1 & 1 & 0 & 1 \\
\hline $\begin{array}{c}\text { Tributaria; } \\
\text { Sanitaria }\end{array}$ & 1 & 1 & 1 & 0 & 1 & 1 & 1 \\
\hline $\begin{array}{c}\text { Laboral; } \\
\text { Agrope- } \\
\text { cuaria }\end{array}$ & 1 & 1 & 1 & 1 & 1 & 1 & 1 \\
\hline Defensa & 0 & 1 & 0 & 0 & 0 & 0 & 0 \\
\hline Vivienda & 1 & 0 & 1 & 0 & 0 & 1 & 0 \\
\hline Educación & 0 & 1 & 0 & 0 & 1 & 0 & 0 \\
\hline
\end{tabular}

Fuente: Elaboración propia, según encuesta a expertos, 2017.

Hasta aquí tenemos tres configuraciones que explican las importantes innovaciones en las políticas públicas con cambios de tercer grado y por otro lado, tres configuraciones que explicarían los más modestos cambios de primer orden (Cuadro 6).

Las combinaciones de las filas 2 y 3 pueden ser simplificadas. Por lo cual podemos presentar nuestras soluciones para $\mathrm{R}=1$, o sea cambios de tercer orden, de esta manera:

$\mathrm{R}=\mathrm{ORG}{ }^{\star} \mathrm{COOR}{ }^{\star}$ moni ${ }^{\star} \mathrm{APOL}{ }^{\star} \mathrm{PART}^{\star}{ }^{\text {cont }}+\mathrm{OR} \mathrm{O}^{\star} \mathrm{COOR}^{\star} \mathrm{MONI} \mathrm{PAR}$ $\mathrm{T}^{\star} \mathrm{CONT}=\mathrm{OR} \mathrm{G}^{\star} \mathrm{COOR}{ }^{\star} \mathrm{PART}\left(\mathrm{moni}{ }^{\star} \mathrm{APOL}+\mathrm{MONI}\right)=1$

De otra manera, se registra un cambio de tercer orden en las políticas públicas cuando se desarrollan tanto capacidades técnico-administrativas como capacidades estatales político-administrativas. 
En una configuración contamos con la presencia de dos capacidades técnico-administrativas (la presencia de las burocracias gubernamentales profesionalizadas y el funcionamiento de mecanismos de coordinación intra-gubernamental), en ausencia de procedimientos de monitoreo, y con la presencia de dos capacidades político-relacionales (interacciones institucionalizadas entre actores burocráticos y agentes parlamentarios de diversos partidos, y existencia y funcionamiento de mecanismos de participación social). Este es el caso de la educación superior.

En la otra configuración, para obtener cambios de tercer orden se requiere la presencia de las tres capacidades técnico administrativas, a lo que hay que agregar la presencia de las capacidades político-relacionales de la participación, y la fiscalización por parte de agencias de control.

Para registrar cambios de tercer orden se precisa como condición necesaria la presencia de dos capacidades técnico-administrativas: el fortalecimiento de burocracias gubernamentales profesionalizadas, y el funcionamiento de mecanismos de coordinación. A las que debe adicionarse una capacidad político-relacional: la existencia de mecanismos de participación social. Siendo las condiciones suficientes, en un caso, la sola presencia de procedimientos de monitoreo; y en el otro, la presencia de interacciones institucionalizadas entre actores burocráticos y agentes políticos parlamentarios de diversos partidos, más la ausencia de procedimientos de monitoreo.

Las combinaciones de las filas 6 y 8 del Cuadro 5 pueden ser simplificadas. Por lo cual podemos presentar nuestras soluciones para $\mathrm{R}=0=$ cambios de primer orden, de esta manera:

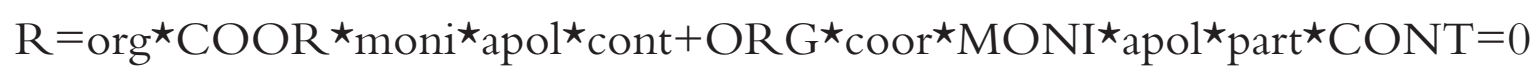

Por ende, los cambios de primer orden están asociados a un escaso desarrollo de las capacidades técnico-administrativas y de las capacidades político-relacionales.

En una configuración que coincide con los casos de las políticas de defensa y de educación, la única capacidad estatal presente es técnica-administrativa, y es la coordinación intra-gubernamental.

La otra configuración con el mismo resultado -esto es, cambio de primer orden o mera recalibración- es el caso de la política de vivienda. Este presenta dos capacidades técnico-administrativas (fortalecimiento de las burocracias gubernamentales profesionalizadas y monitoreo) y una político-relacional (la fiscalización de agencias de control).

\section{A modo de conclusión: capacidades estatales y tipo de innovación de las políticas del decenio progresista uruguayo}

En este trabajo nos hemos preguntado si las diferentes políticas implementadas durante los primeros diez años de gobiernos nacionales del Frente Amplio han supuesto 
cambios en los contenidos de las políticas públicas imperantes hasta marzo del año 2005, y cómo han incidido las capacidades estatales en ese resultado. Aunque corresponde advertir que en nuestro estudio no se incluyeron políticas tan relevantes como las macroeconómicas, de energía o de reforma de la administración pública, el amplio elenco de políticas seleccionado puede dar cuenta de la tónica predominante del policymaking de la izquierda uruguaya.

En primer término, del análisis presentado deriva una nota de interés para el análisis del caso, y eventualmente como manifestación específica de fenómenos más generales de interés académico: la relación entre partidos de izquierda, sus ideologías y las capacidades estatales desarrolladas en las diferentes políticas públicas, por una parte, y las características de las políticas públicas diseñadas, por otra. En el caso estudiado, el gobierno nacional ejercido por un partido de izquierda fue capaz de alterar patrones previos de políticas en los sectores en los que contó - o pudo desarrollar- capacidades estatales significativas.

Como consecuencia, la intensidad de los cambios introducidos en las políticas ha sido marginal en algunas arenas; pero adquirió una dimensión mucho mayor en otras. Esta comprobación abona las teorías que recientemente han revalorizado el potencial transformador de las estrategias gradualistas (Baumgartner et al, 2006; Pierson, 2004; Streek\&Thelen, 2005), que se demuestran también válidas para sistemas como el uruguayo, en los que el proceso de negociación adquiere una densidad apreciable.

La constatación de que esas innovaciones convivieron con la inercia o el bloqueo de otras iniciativas de políticas (en la educación básica, en la defensa nacional, en las políticas de vivienda) hace manifiesta la importancia de las variables específicas de cada una de las arenas de políticas, capaces de conducir a resultados diversos aún ante estrategias similares (Marsh; Rhodes, 1992). Entre esas variables se hallan las capacidades estatales. Estudios anteriores han ubicado a Uruguay entre los países de América Latina que evidencian un mayor nivel de desarrollo de sus capacidades estatales (Franco Chuaire; Scartascini, 2014; Luna;Toro, 2014; World Bank, 2015). Precisando estos asertos, nuestra indagación permite afirmar que el desarrollo diferencial de dichas capacidades en distintos puntos del entramado estatal se asoció con la aptitud del estado uruguayo para promover transformaciones de mayor o menor magnitud.

Más específicamente, como resultado del análisis descriptivo de las capacidades técnico-administrativas y político-relacionales, y del análisis comparativo cualitativo de las mismas capacidades en trece políticas públicas en el aquí llamado "decenio progresista" uruguayo, podemos afirmar:

a) Las capacidades técnico-administrativas se desarrollaron algo más que las político-relacionales.

b) La presencia de cambios de tercer orden en las políticas públicas progresistas está asociado al desarrollo de capacidades tanto técnico-administrativas como político-relacionales. 
c) Los cambios de primer orden en las políticas públicas progresistas están asociadas a un escaso desarrollo de capacidades estatales.

En suma: en el caso uruguayo, las capacidades estatales resultan variables relevantes para contribuir a comprender las transformaciones en las políticas públicas, resultando de particular significación la constatación de que en los sectores en que dichas capacidades fueron fuertes, fue posible propiciar transformaciones de porte, categorizadas aquí como cambios de tercer grado; y por el contrario, donde las capacidades fueron débiles, los cambios en las políticas tuvieron escasa relevancia, o de primer grado. Esta constatación, válida para el caso y el período estudiado, debería ponerse a prueba en otros para discutir las condiciones de su generalización.

\section{Referencias}

ALTMAN, David; LUNA, Pablo. Introducción: el estado latinoamericano en su laberinto. Revista de Ciencia Política,2012, p. 521-543,

ANTIA, Florencia. La política tributaria durante el gobierno de Mujica: ¿hacia el fin del secreto bancario?.En: BENTANCUR, Nicolás.; BUSQUETS, José Miguel (Coord.).El decenio progresista. Las Políticas Públicas, de Vázquez a Mujica. Montevideo: ICP- Fin de Siglo, 2016.

BAUMGARTNER, Frank R; GREEN-PEDERSEN, Christoffer; JONES, Bryan D. Comparative studies of policy agendas. Journal of European Public Policy, v. 13, n. 7, 2006.

BENTANCUR, Nicolás; BUSQUETS, José Miguel (Coord.). El decenio progresista. Las Políticas Públicas, de Vázquez a Mujica. Montevideo: ICP- Fin de Siglo, 2016.

; CLAVIJO, Emiliano. La educación superior durante el decenio frenteamplista: análisis de un caso de innovación de políticas. En: BENTANCUR, Nicolás; BUSQUETS, José Miguel (Coord.). El decenio progresista. Las Políticas Públicas, de Vázquez a Mujica. Montevideo: ICP- Fin de Siglo, 2016.

BENTANCUR, Nicolás;MANCEBO, María Ester.El discreto encanto del gradualismo. Continuidad, restauración e innovación del primer gobierno de izquierda. En: MANCEBO, Maria Ester.; NARBONDO, Pedro (Coord.), Reforma del Estado y políticas públicas de la Administración Vázquez: acumulaciones, conflictos y desafios. Montevideo: Fin de Siglo, 2010.

BUSQUETS, José Miguel; SCHENCK, Marcela. La política pública de seguridad social en el gobierno del presidente Mujica: novedades y frenos. En: BENTANCUR, Nicolás.; BUSQUETS, José Miguel (Coord.). El decenio progresista. Las Políticas Públicas, de Vázquez a Mujica. Montevideo: ICP- Fin de Siglo, 2016.

CAETANO, Gerardo; PEREZ, Romeo; RILA, José. La partidocracia uruguaya: historia y teoría de la centralidad de los partidos políticos. Cuadernos del CLAEH. 44, p. 37-62, 1987.

; LOPEZ, Camilo; LUJAN, Carlos. La política exterior uruguaya entre Vázquez y Vázquez (2010 \ 2015). En: BENTANCUR, Nicolás.; BUSQUETS, José Miguel (Coord.). El decenio progresista. Las Políticas Públicas, de Vázquez a Mujica.Montevideo: ICP- Fin de Siglo, 2016.

CAPOCCIA, Giovanni; KELEMEN, R. Daniel.The Study of Critical Junctures: Theory Narrative, and Counterfactuals in Historical Institutionalism. World Politics, n. 59, 2007, p. 341-369.

CAMPBELL,John L.;PEDERSEN, Ove.Knowledge Regimes and Comparative Political Economy.En:BÉLAND, Daniel ; COX, Robert Henry (Ed.). Ideas and Politics in Social Science Research.Oxford: Oxford UniversityPress, 2011. 
CARRACEDO, Fabián; SENATORE, Luis. Las políticas laborales y las relaciones de trabajo durante el gobierno de José Mujica. En: BENTANCUR, Nicolás.; BUSQUETS, José Miguel (Coord.). El decenio progresista. Las Políticas Públicas, de Vázquez a Mujica. Montevideo: ICP- Fin de Siglo, 2016.

CECCHINI, Simone; MARTINEZ, Rodrigo. Protección social inclusiva en América Latina. Una mirada integral, un enfoque de derechos. Santiago: CEPAL, 2012.

CHASQUETTI, Daniel.Actividad del Poder Legislativo. En: CAETANO, Gerardo (Coord.).Informe de Coyuntura $N^{o}$ 10. Política en tiempos de Mujica. En busca del rumbo. Montevideo: ICP- Estuario, 2010.

Estudio sobre la actividad del parlamento. $47^{\circ}$ Legislatura.2014. Disponible en: < http://cienciassociales. edu.uy/wp-content/uploads/2014/09/Chasquetti.pdf>. 30 nov. 2015.

COLLIER, Ruth; COLLIER, David. Shaping the political arena: critical junctures, the Labor movement and regime dynamics in Latin America.Princeton: Princeton UniversityPress, 1991.

CRUZ-RUBIO, César Nicandro. La taxonomía del cambio: enfoques y tipologías para la determinación del cambio de las políticas públicas. Andamios, v. 9, n. 20, 2012. p. 297-321.

DE RIZ, Liliana. Uruguay: la política del compromiso. En: AAVV, Cultura Política y alternancia en América Latina. Madrid: Editorial Pablo Iglesias, 2008.

DINIZ, Eli. Crise, Reforma do Estado e Governabilidade. Rio de Janeiro: Editora da Fundação Getúlio Vargas, 1997.

DURANT, Robert F; DIEHL, Paul F. Agendas, alternatives and public policy: lessons from the U.S. foreign policy arena. Journal of Public Policy,v. 9, n. 2, 1989, p. 179-205,.

EDIGHEJI, O. Constructing the 21 st century development State:Potencials and pifalls. Cape City: HRSC Press, 2010.

EVANS, P.; RAUCH, J. Bureucracy and Growth: A Cross-National Analysis of the Effects of "Weberian" State Structures on Economic Growth, American Sociological Review, v. 64, n.5, 1999, p. 748-765.

FILGUEIRA, Fernando. El nuevo modelo de prestaciones sociales en América Latina: residualismo, eficiencia y ciudadanía estratificada. En: ROBERTS, Brian (Ed.).Ciudadanía y Políticas Sociales. San José de Costa Rica: FLACSO/SSRC, 1998.

FRANCO, María Chuaire; SCARTASCINI, Carlos. La política de las políticas públicas: Re-examinando la calidad de las políticas públicas y las capacidades del Estado en América Latina y el Caribe. Resumen de Políticas del BID, v. 220, 2014.

FUKUYAMA, Francis. La construcción del Estado. Hacia un nuevo orden mundial en el Siglo XXI. Barcelona: Ediciones B, 2004.

GARCE, Adolfo. Regímenes Políticos de Conocimiento: construyendo un nuevo concepto a partir de eventos de cambio seleccionados en políticas públicas del gobierno de Tabaré Vázquez (Uruguay, 2005-2009). Revista de Ciencia Política,v. 34, n. 2, 2014, p. 439-458..

GARCÍA - PELAYO, Manuel. El Estado de partidos. Madrid:Alianza Editorial, 1986.

GUEDDES, Barbara. Reform as a Collective Good. En: Politician's Dilemma: Building State Capacity in Latin America. Berkeley: University of California Press, 1994. p. 24-42.

GONZALEZ, Julián. La cuestión militar: luego de cinco años llegan los desafíos más complejos. En: MANCEBO, Maria. Ester; NARBONDO, Pedro (Coord.), Reforma del Estado y políticas públicas de la Administración Vázquez: acumulaciones, conflictos y desafíos. Montevideo: Fin de Siglo, 2010.

GONZALEZ GUYER, Julián; MENDEZ BARBATO, Gustavo. La Defensa Nacional en tiempos de Mujica. De un Tímido Impulso y su Freno. En: BENTANCUR, Nicolás; BUSQUETS, José Miguel (Coord.).El decenio progresista. Las Políticas Públicas, de Vázquez a Mujica. Montevideo: ICP- Fin de Siglo, 2016.

GRINDLE, Merilee S. and John W.Thomas.Public Choices And Policy Change: The Political Economy Of Reform In Developing Countries. Baltimore: John Hopkins University Press, 1991. 
HALL, Peter A. (Ed.). The Political Power of Economic Ideas: Keynesianism across Nations. Princeton: Princeton University Press, 1989.

Policy Paradigms, Social Learning, and the State: The Case of Economic Policymaking in Britain. Comparative Politics, v. 25, n. 3, 1993, p. 275-296.

HART, Paul't. Understanding Public Leadership.Londres: Palgrave Macmillan, 2014.

HENDRIX, Cullen. Measuring state capacity: Theoretical and empirical implications for the study of civil conflict. Journal of Peace Research, 2010, p.273-285.

HOGWOOD, Brian; PETERS, B. G. The Dynamics of Policy Change: Policy Succession. Policy Sciences, v. 14, n. 3, 1982, p.225-245.

HOOD, Christopher. A public management for all seasons?.Public Administration, n. 69, 1991.

HOWLETT, Michael; CASHORE, Benjamin. The Dependent Variable Problem in the Study of Policy Change: Understanding Policy Change as a Methodological Problem. Journal of Comparative Policy Analysis, v. 11, n. 1, 2009, p. 33-46.

HUBER, Evelyn; STEPHENS, J. D. The political economy of pension reform: Latin America in comparative perspective. Geneva OccasionalPaper, n. 7, UNSRID, 2000.

IAZZETTA, Osvaldo. Capacidades estatales, gobernabilidad democrática y crisis global. Crisis Global $y$ Gobernabilidad en América Latina, Buenos Aires: PNUD/Siglo XXI, 2012.

JOHNSON, Niki; SEMPOL, Diego. Igualdad de género y derechos de la diversidad sexual: avances y rezagos en el gobierno de Mujica. En: BENTANCUR, Nicolás.; BUSQUETS, José Miguel (Coord.). El decenio progresista. Las Políticas Públicas, de Vázquez a Mujica. Montevideo: ICP- Fin de Siglo, 2016.

LANE, Jan-Erik. The public sector.Concepts, models and approaches. London: Sage, 1995.

LUNA, Juan Pablo; TORO MAUREIRA, Sergio. Capacidad del Estado y gobernabilidad democrática en América Latina: un acercamiento de medición y evaluación basado en el Barómetro de las Américas. Perspectivas desde el Barómetro de las Américas, v. 102, 2014.

LANZARO, Jorge. Sindicatos y sistema político. Relaciones corporativas en el Uruguay 1940-1985. Montevideo: FCU, 1986.

Continuidad y cambios en una vieja democracia de partidos. Uruguay 1910-2010. Opiniao Pública, v. 19, n. 2, 2013, p. 235-269.

LINDBLOM, Charles. La ciencia de "salir del paso". En:VILLANUEVA, L.Aguilar (Ed.).La hechura de las politicas. México: Porrúa, 1992. p. 201-225.

LYNN, Laurence E. Public Management: Old and New. Nueva York: Routledge, 2006.

MAGRI, Altair. La vivienda: una nave insignia en aguas turbulentas. Proceso y derivas durante el quinquenio de José Mujica. En: BENTANCUR, Nicolás; BUSQUETS, José Miguel (Coord.). El decenio progresista. Las Políticas Públicas, de Vázquez a Mujica. Montevideo: ICP- Fin de Siglo, 2016.

MAHONEY, James; THELEN, Kathleen.Explaining institutional change.Ambiguity, agency and power.Cambridge: Cambridge University Press, 2010.

MANCEBO, María Ester; LIZBONA, Alexandra. El statu quo en la educación obligatoria: entre la partidocracia, los sindicatos y el fantasma de la Reforma Rama. En: BENTANCUR, Nicolás; BUSQUETS, José Miguel (Coord.). El decenio progresista. Las Políticas Públicas, de Vázquez a Mujica. Montevideo: ICP- Fin de Siglo, 2016.

MANN, Michael. The autonomous Power of the State: Its Origins, Mechanisms and Results. European Journal of Sociology, v. 25,1984, p. 185-213.

MARTINEZ, Juliana. Regímenes de Bienestar en América Latina. Madrid: Fundación Carolina, 2007.

MARSH, David; RHODES, Roderick Arthur Willian. Policy Networks in British Government.Oxford: ClarendonPress, 1992. 
MIDAGLIA, Carmen. Un balance crítico de los programas sociales en América Latina. Entre el liberalismo y el retorno del Estado. Nueva Sociedad, v. 239, 2012, p. 79-89.

MIDAGLIA, Carmen; TEJERA, Rafael. Uruguay del Pepe Mujica: los cambios procesados en el campo de la asistencia social. En: BENTANCUR, Nicolás; BUSQUETS, José Miguel (Coord.). El decenio progresista. Las Políticas Públicas, de Vázquez a Mujica. Montevideo: ICP- Fin de Siglo, 2016.

NARBONDO, Pedro. Contratos gerencialistas, estado neoliberal y autonomía enraizada. Revista Uruguaya de Ciencia Política, v. 20, n. 1, ,2011, p. 1-31..

OFFE, Claus. Governance: an “empty signifier”?.Constellations, v. 16, 2009, p. 550-562,.

OSZLAK, Oscar. Políticas públicas y regímenes políticos: Reflexiones a partir de algunas experiencias latinoamericanas. Estudios CEDES,1980.

PEREIRA, Javier. Hacia una nueva agenda en el relacionamiento Estado-Sociedad Civil en Uruguay.Documento 14.InstitutoHumanista Cristiano Juan Pablo Terra, 2015.

PIERSON, Paul. Increasing Returns, Path Dependence, and the Study of Politics. The American Political Science Review ,v. 94, n. 2, 2000, p. 251-267.

Politics in Time: History, institutions, and social analysis.Princeton: Princeton University Press, 2004.

PIRES, Roberto; GOMIDE, Alexandre. Governanca e capacidades estatais: uma análise comparativa de programas federais. Revista de Sociología e Política, 2016, p. 121-143.

POLLITT, Christopher; BOUCKAERT, Geert. Public Management Reform. Oxford: Oxford University Press, 2004.

PRESTON, Thomas; HART, Paul 't. Understanding and Evaluating Bureaucratic Politics: The Nexus between Political Leaders and Advisory Systems. Political Psychology, v. 20, n. 1, 1999, p. 49.98.

REPETTO, Fabián. Capacidad Estatal: requisito para el mejoramiento de la política social en América Latina. Documento de Trabajo del INDES, BID, 2004.

SABATIER, Paul A.; WEIBLE, Christopher M. El marco de las coaliciones promotoras. Innovaciones y clarificaciones. En: SABATIER, Paul A. (Ed.). Teorías del proceso de las políticas públicas. Buenos Aires: Jefatura de Gabinete de Ministros de la Presidencia de la Nación, 2010.

SCHMITTER, Philippe. Modes of interest intermediation and models of societal change in Western Europe. Jornal Comparative Political Studies, Sage Publications, 1977.

SETARO, Marcelo. Políticas de Salud durante el Gobierno de José Mujica. Avances en el Sistema Nacional Integrado de Salud y perspectivas frente a una era de austeridad. En: BENTANCUR, Nicolás; BUSQUETS, José Miguel (Coord.). El decenio progresista. Las Políticas Públicas, de Vázquez a Mujica. Montevideo: ICP- Fin de Siglo, 2016.

SOIFER, Hillel David. Measuring state capacity in contemporary Latin America.Revista de Ciencia Política,v. 32, n. 3, 2012, p. 585-598.

STREECK, Wolfgang;THELEN, Kathleen.Beyond continuity. Institutional change in advanced political economies. New York: Oxford University Press, 2005.

STEWART, Jenny.Value Conflict and Policy Change. The Review of Policy Research, v. 23, n. 1, 2006, p. 183-195.

TILLY, Charles. Coerción, capital y los Estados europeos, 990-1990. Madrid: Alizanza Editorial, 1992.

VICHER, Diana. La reforma neogerencial en Nueva Zelanda. Convergencia,v.14, n. 45, 2007, p. 163-185.

VILA, Alejandro. La política de Seguridad Ciudadana del mujiquismo: entre acuerdos y disensos. En: BENTANCUR, Nicolás; BUSQUETS, José Miguel (Coord.). El decenio progresista. Las Políticas Públicas, de Vázquez a Mujica. Montevideo: ICP- Fin de Siglo, 2016.

WEIR, Margaret; SKOCPOL, Theda. Las estructuras del Estado: una respuesta "keynesiana" a la Gran Depresión. Zona Abierta 63/64, 1993, p. 73-153. 
WORLD BANK. Worldwide Governance Indicators.Disponible en: < http://info.worldbank.org/governance/ wgi/index.aspx\#home>. 15 jun. 2017

ZURBRIGGEN, Cristina. Uruguay, un país “Agro Inteligente”.En: BENTANCUR, Nicolás; BUSQUETS, José Miguel (Coord.). El decenio progresista. Las Políticas Públicas, de Vázquez a Mujica. Montevideo: ICP- Fin de Siglo, 2016. 


\section{ANEXO I: Notas metodológicas}

Se sintetizan a continuación los pasos necesarios para la aplicación del método QCA.

Tomadas de la teoría o del marco analítico las condiciones causales y los resultados, se presentarán de forma dicotómica, dando el valor de uno a la presencia de la causa o resultado y de cero a la ausencia de los mismos. Los criterios para realizar la dicotomización son arbitrarios o están informados por la teoría. Con esta información se construye la tabla de la verdad, la que será minimizada de forma de alcanzar una expresión sencilla, de fácil interpretación. Cada fila de la tabla representa una combinación y cuando dos o más casos tienen la misma combinación pasan a ser representados por una sola fila, por lo que cada fila puede contener uno o muchos casos.

La minimización sigue tres pasos: los ceros (ausencias) y unos (presencias) son sustituidos por las letras minúsculas y mayúsculas correspondientes; las dos combinaciones o filas de la tabla de la verdad que sólo difieren en una condición se resumen en una sola combinación, contando con todas las condiciones que son iguales en ambas combinaciones (el procedimiento se repite todas las veces posibles); y a las expresiones resultantes de esas minimizaciones se les denomina "primeros implicantes". Se trata de seleccionar el menor número posible de primeros implicantes.

Por último, así como es posible encontrar diferentes combinaciones causales que lleven al mismo resultado, es probable hallar combinaciones iguales con resultados diferentes. Estas últimas, son las llamadas contradicciones. El número de combinaciones contradictorias es un buen indicador del grado de eficiencia de la teoría. Las contradicciones, luego de encontradas, pueden ser resueltas. La forma más recomendable es estudiar la selección de variables realizada de forma de llegar a resultados no contradictorios. Concordemos que esta es sin duda una de las grandes virtudes del enfoque booleano.

Luego de seguido este procedimiento podemos llegar a una explicación en donde varias y diferentes combinaciones puedan conducir a un mismo resultado y donde importan tanto la ausencia como la presencia. 


\section{ANEXO II: Expertos consultados}

Antía, Florencia (Tributaria)

Bentancur, Nicolás (Educación Superior)

Busquets, José Miguel (Seguridad Social)

Carracedo, Fabián (Laboral)

González, Julián (Defensa)

Lizbona, Alexandra (Educación)

Lopez, Camilo (Exterior)

Luján, Carlos (Exterior)

Magri, Altair (Vivienda)

Mancebo, Ester (Educación)

Schenck, Marcela (Seguridad Social)

Setaro, Marcelo (Salud)

Tejera, Rafael (Asistencia Social)

Vila, Alejandro (Seguridad Pública)

Zurbriggen, Cristina (Agropecuaria) 


\title{
State capacities and change in public policies: The case of Uruguay under the governments of the FrenteAmplio (2005-2015)
}

\begin{abstract}
The article aims to investigate the change in public policies in Uruguay during the leftist governments of the FrenteAmplio (2005-2015). In order to do so, it analyzes how public policies have changed, how they did it and the associations that can be established between the different state capacities developed and the type of change implemented in public policies of the progressive Uruguayan decade (2005-2015). After an inquiry that rests on previous studies, a consultation to experts and the application of the QCA methodology concludes that in Uruguay, the arrival in the executive branch of a leftist party has led to significant changes in the form and substance of policies, although with unequal intensity in different points of the public tissue. The state capacities were relevant variables to help understand these transformations, resulting in particular significance the finding that in sectors where these capacities were strong, it was possible to promote transformations of size and where they were not, there were minor changes.
\end{abstract}

Keywords: public Policies, left Governments, state capacities, change in public policies, Uruguay.

\section{As capacidades do Estado e mudança de políticas públicas: 0 caso do Uruguai sob os governos da Frente Ampla (2005-2015)}

\section{Resumo}

O trabalho tem como objetivo fazer indagações sobre as transformações nas políticas públicas no Uruguai durante os governos de esquerda, especificamente, o partido Frente Ampla (20052015). Para isso, se analisa a intensidade das transformações em um conjunto de políticas e quais as associações existentes entre as diferentes capacidades desenvolvidas e o tipo de transformação implementado por cada uma delas. Após uma pesquisa que se baseia em trabalhos prévios, em uma consulta a especialistas e na implementação da metodologia análise qualitativa comparativa, conclui-se que, embora com intensidade variável em diferentes partes do tecido público, no Uruguai a chegada ao poder executivo do Frente Ampla trouxe mudanças significativas na forma e direcionamento das políticas. Para contribuir à compreensão dessas transformações, as capacidades do Estado se apresentam de diversas formas, demonstrando que nos setores em que essas capacidades eram fortes, foi possível realizar transformações relevantes e onde as capacidades foram fracas se registraram transformações de menor intensidade.

Palavras-chave: políticas públicas, governos de esquerda, capacidades estatais, mudanças nas políticas públicas, Uruguai. 